\title{
Effect of Continuous Skin Temperature Monitoring on Nursing Care for Patients After Flap Transplantation for Hand Skin Defect Repair
}

\author{
Wenxian Liang', Qingran Lin ${ }^{2}$, *, Weiju Chen ${ }^{3}$, Yuxia Guan ${ }^{1}$, Meiling He ${ }^{1}$, Kelie Wang ${ }^{1}$, \\ Yanjun Yang ${ }^{1}$, Ziqing Zhang ${ }^{1}$ \\ ${ }^{1}$ Longgang Orthopedics Hospital of Shenzhen, Shenzhen, China \\ ${ }^{2}$ The First Affiliated Hospital of Jinan University, Guangzhou, China \\ ${ }^{3}$ School of Nursing of Jinan University, Guangzhou, China
}

Email address:

qingranlin@126.com (Qingran Lin)

${ }^{*}$ Corresponding author

\section{To cite this article:}

Wenxian Liang, Qingran Lin, Weiju Chen, Yuxia Guan, Meiling He, Kelie Wang, Yanjun Yang, Ziqing Zhang. Effect of Continuous Skin Temperature Monitoring on Nursing Care for Patients After Flap Transplantation for Hand Skin Defect Repair. International Journal of Systems Engineering. Vol. 4, No. 2, 2020, pp. 12-17. doi: 10.11648/j.ijse.20200402.11

Received: June 15, 2020; Accepted: June 28, 2020; Published: July 13, 2020

\begin{abstract}
Objective: The article aims to explore the effect of continuous skin temperature monitoring on nursing for flap transplantation for repairing hand skin soft tissue defect. Methods: We selected 70 patients who received flap transplantation for hand skin soft tissue defect repair from April 2016 to April 2018 in a hospital and averagely randomized them into control group and observation group. Both groups were given routine nursing and the observation group also received continuous skin temperature monitoring. The incidence of vascular crisis, length of stay and duration of wound healing were compared between the two groups. Results: The incidence of vascular crisis, length of stay and duration of wound healing of the observation group were significantly lower than those of the control group $(\mathrm{P}<0.05)$. Conclusions: Continuous skin temperature monitoring has a great effect on the nursing for patients who received flap transplantation for hand skin defect repair. It can help detect circulatory crisis earlier so that medical staff can give intervention earlier, which can effectively reduce occurrence of complications of vascular crisis and thus is worth clinical application.
\end{abstract}

Keywords: Skin Temperature Monitoring, Flap Transplantation, Repair, Hand Skin Defect

\section{Introduction}

In recent years, with the development of the society, various traffic accidents and accidental injuries cause an increase in the occurrence of hand injury which may lead to skin soft tissue defect or even bone exposure, making clinic treatment more difficult [1]. Application of flap in repairing hand skin soft tissue defect can effectively improve the shape of the damaged skin with high flap survival rate and less postoperative complications. Currently, it is the major method to repair the skin soft tissue defects of the limbs [2]. However, it is necessary to measure the flap surface temperature of the patient after surgery, which is conducive to knowing about the blood flow of the repaired part. The effectiveness and sensitivity of the skin thermometer to monitor the skin temperature are very high, and application of skin temperature monitoring to the skin flap repairing skin soft tissue defects of the hand is of significant effect [3]. We adopted continuous skin temperature monitoring in nursing patients who had flap transplantation to repair skin soft tissue defect of hand, which brought satisfactory results. The research is reported as follows.

\section{Data and Methods}

\subsection{General Data}

The research included 70 patients with hand skin soft tissue defect, admitted to our hospital from April 2016 to April 2018 
and randomized them into two groups: control group (35 cases) and observation group (35 cases). Both groups received routine nursing and the observation group was also given continuous skin temperature monitoring. The incidence of vascular crisis, length of stay and duration of wound healing were observed and compared between the two groups. The inclusion criteria were: (1) all patients meeting the criteria for skin flap repairing skin soft tissue defects; (2) patients with normal vital signs; (3) patients without other major diseases; (4) patients agreeing to the study; (5) the granulation tissue growing well after the first-stage treatment of the wound surface with negative bacterial culture. Exclusion criteria were: (1) patients with soft tissue defects in other parts; (2) patients with ambiguous consciousness; (3) patients with unstable vital signs; (4) patients with major diseases. In the control group, there were 15 cases combined with other injuries (in blood vessel, nerve and tendon) and 20 cases of pure injury of blood vessel, with defect area between $6 \mathrm{~cm}^{2}$ and $186 \mathrm{~cm}^{2}$. In the observation group, there were 14 cases combined with other injuries (in blood vessel, nerve and tendon) and 21 cases of pure injury of blood vessel with defect area between $5 \mathrm{~cm}^{2}$ and $204 \mathrm{~cm}^{2}$.

\subsection{Methods}

\subsubsection{Control Group}

Patients in control group received routine nursing care for flap transplantation, including: (1) Preoperative mental nursing: the nursing staff should explain the main operation method, purpose and importance to the patients in easy-to-understand language, so that the patients can fully understand the matters needing attention, improving their compliance. Besides, nursing staff should maintain good communication with patients, and reduce the inner pressure of patients through appropriate methods. (2) Nursing for the surface of the wound: blood drawing and infusion in skin donor site and injury area were not allowed. One day before the operation, angiography was used to detect the arteries in the donor area and Doppler ultrasound was used to check the perforator vessels [4]. ADF 0.5\% PVP-I disinfectant was used for skin disinfection and aseptic towels to wrap the skin. Skin preparation was performed on the day of the operation, and the patient's fingernails were cut short. (3) After the operation, the room temperature was maintained at $23^{\circ} \mathrm{C}-25^{\circ} \mathrm{C}$, and the humidity at $50 \%-65 \%$. The flap was continuously irradiated with a $60 \mathrm{~W}$ heating lamp, and the heating lamp was about 30 to $40 \mathrm{~cm}$ away from the flap [5]. The skin temperature of the affected limb was the same as or slightly higher than the normal skin temperature by 1.00 to $2.00^{\circ} \mathrm{C}$. If the skin temperature of the patient was 3.00 to $4.00^{\circ} \mathrm{C}$ lower than the normal skin temperature, it indicated that there was a vascular crisis, and the doctor should be notified in time for treatment. (4) Nursing for skin flap: the nurse observed the wound surface after skin flap transplantation to keep the drainage smooth. The blood circulation of the flap as observed every 30 to 60 minutes within 24 hours after the operation, including observing and recording the capillary reaction, tissue tension, temperature, color, etc. It should be gentle to observe the blood vessel at the flap in order to avoid damage to the flap. If any abnormality was found, the nurse should immediately notify the doctor for treatment. Close observation was required 3 days after the operation. If there was no abnormality, the observation could be done every 4 to 6 hours. (5) Pain nursing: the analgesics were given three days before the operation, which was to reduce the vasospasm caused by pain because it would affect the blood supply to the transplanted flap. (6) Discharge instruction: continuing nursing staff provided health education for the patients before they were discharged from the hospital. After the wounds healed, the nurse instructed the patients to gradually take functional exercises. The nursing staff also evaluated patients' living environment, guided their rehabilitation training, and made an appointment with the patients for home continuing care after their discharge.

\subsubsection{Observation Group}

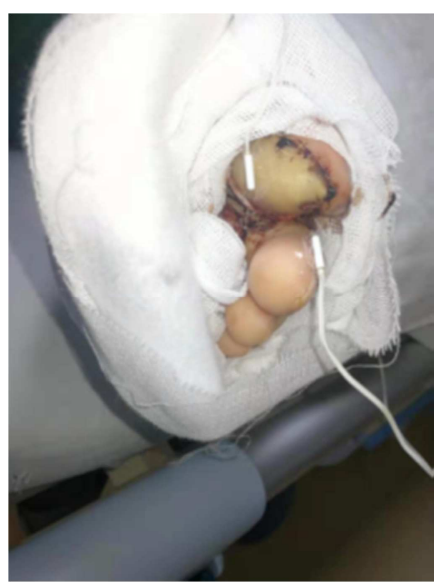

Figure 1. The probe of the skin temperature monitor.

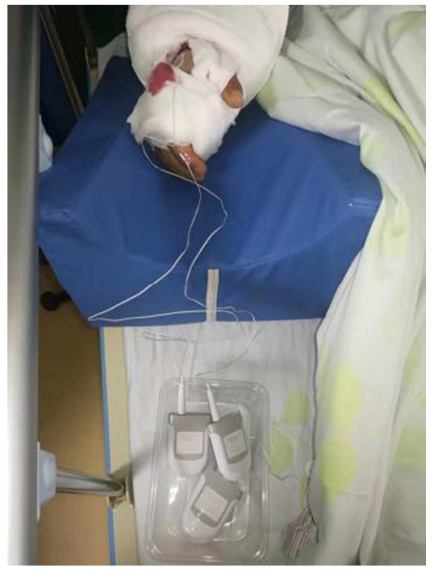

Figure 2. The probe of the skin temperature monitor.

Besides routine nursing care for flap transplantation, continuous skin temperature was also given to the observation group, including the following measures: (1) A company's $\mathrm{MH}-70 \mathrm{~N}$ skin temperature monitor was used. The skin temperature value measured by the thermistor of the monitor was transferred to the central skin temperature management system via Bluetooth to WIFI, and the patient's skin 
temperature data were collected, analyzed, stored through the functional map manager, which could assist medical staff to observe skin temperature changes and determine whether the blood flow fitted the blood vessel. When the monitored skin temperature was lower than $33.00^{\circ} \mathrm{C}$ or higher than $35.00^{\circ} \mathrm{C}$, there would be an alarm for medical staff to pay attention to the situation of patient's flap, and at the same time skin temperature curve on the display screen widened to give a visual remind. (2) Three days after the operation, strict attention was paid to the skin temperature, blood flow, tension, capillary reaction and other conditions of the transplanted flap.
When using the skin temperature monitor, the position of the probe should be changed every 1 hour to avoid local pressure for too long. At the same time, change of position can also monitor skin temperature of different parts to know about the temperature of the transplanted skin flap more clearly. The probe of the skin temperature monitor was fixed with transparent applicator (see Figure 1 and Figure 2), and the measurement site was marked. The skin temperature of the site was displayed in the computer with a wavy line (see Figure 3).

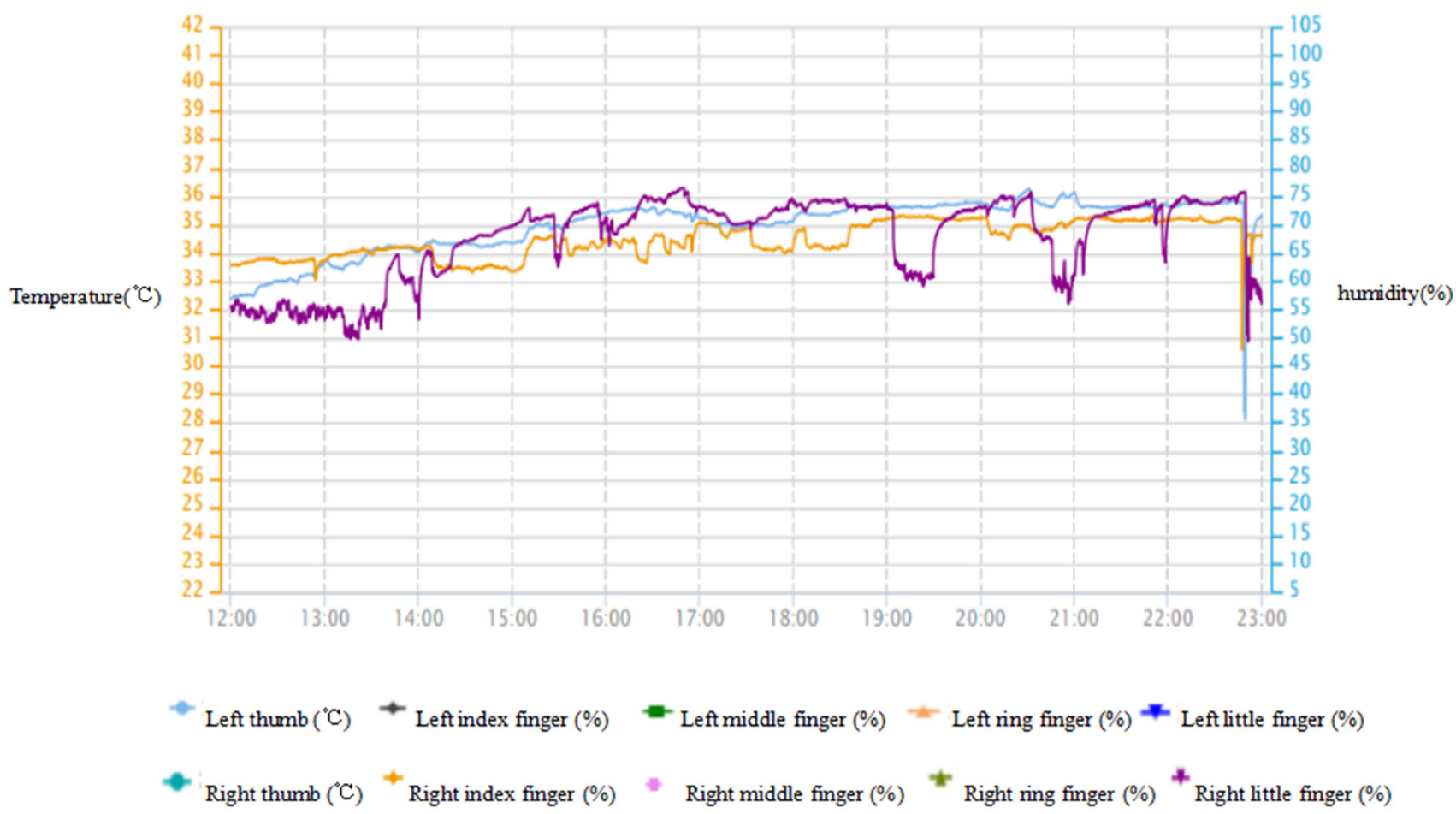

Figure 3. The skin temperature of the site was displayed in the computer.

(3) Continuous monitoring for 7 days after surgery showed the flap temperature. When the skin temperature fluctuated greatly, attention should be paid to the flap situation in time to eliminate external causes, such as change of the probe, too low or too high room temperature, and find the cause and deal with it in time. (4) To avoid the influence of the heating lamp on the skin temperature monitoring, the healthy side as the control and the part monitored by the skin temperature monitor were placed under the heating lamp. At the same time, the other probe was placed on the healthy finger without exposure to the heating lamp as control. The skin temperatures of the three parts were compared to make the monitored data more reliable. When the skin temperature of the affected limb was the same as the normal skin temperature or there was a difference of $\pm 1.00 \sim 2.00^{\circ} \mathrm{C}$, and the waveform of the skin temperature fluctuation curve was basically the same, it was normal. When the skin temperature of the patient was lower or higher than normal skin temperature by $3.00 \sim 4.00^{\circ} \mathrm{C}$, there was a blood circulation crisis. Close attention should be paid to the situation of the transplanted flap, and the doctor should be promptly notified to deal with it, which should be recorded in real time (as observed: under the heating lamp, the skin temperature of the monitored part and the control part was higher than that of the control part that was not under the heating lamp. If the indoor temperature was $23^{\circ} \mathrm{C}$, the skin temperature of the patient's monitored part and the healthy side would be relatively $1-2^{\circ} \mathrm{C}$ lower. The overall skin temperature changes with the change of the outside temperature. When skin temperature monitoring predicted that a venous crisis might occur, the nursing staff should change local dressing, appropriately raise the local heating lamp, and adopt bloodletting through acupuncture to relieve spasm; if continuous monitoring of skin temperature predicted that an arterial crisis might occur, nursing staff should improve the blood flow of the transplanted flap through increasing the room temperature, local warming, reducing the height of the heating lamp, relieving local wound compression, relieving spasms and pain, anticoagulation, supplying blood volume and other related measures to reduce the incidence of blood circulation crisis, and promote the survival of the transplanted 
flap.

\subsection{Outcome Measurements and Evaluation Standard}

\subsubsection{Outcome Measurements of Postoperative Circulatory Crisis}

The commonly used observation indicators for clinical blood circulation include the following five items: skin temperature, skin color, degree of swelling, capillary filling determination, small incision bleeding or errhysis.

\subsubsection{Evaluation Standard and Treatment for Postoperative Circulatory Crisis}

(1) Regular curve of skin temperature variation: 1) parallel curve: the difference in skin temperature between the transplanted flap and the healthy side is $\pm(0.50-2.00){ }^{\circ} \mathrm{C}$. Parallel change within $0^{\circ} \mathrm{C}$ indicates that the arteriovenous anastomotic opening is smooth, and the blood circulation of the transplanted tissue is good; 2) suddenly lowering curve: when the skin temperature of the transplanted flap and the healthy side suddenly differs by more than $3.00^{\circ} \mathrm{C}$, it is caused by arterial embolism, and surgical exploration should be performed immediately; 3 ) separation curve: the difference in skin temperature between the transplanted flap and the healthy side gradually increases, generally reaching $3^{\circ} \mathrm{C}$ after $24-48$ hours, which is caused by venous embolism; (2) The evaluation criteria and treatment for arterial and venous crisis are as follows: 1) arterial crisis: arterial crisis occurs when skin at the end of the flap is pale or gray, the temperature of the flap is $3^{\circ} \mathrm{C}$ lower than the normal skin temperature, and the skin wrinkles are obviously deepened. Besides, there are also finger pulp deflation without tension, prolonged capillary filling time, weakened or disappeared arterial pulse, no bleeding or slow bleeding at the fingertip incision, dark red color. Once there is arterial crisis, the cause should be first determined. If it is because the vascular pedicle is compressed, the patient's position should be corrected to relieve compression in time. If it is because the vascular pedicle is twisted, antispasmodic and vasodilator drugs should be used. If there is no improvement after 30 minutes, the physician should be notified in time to perform surgical exploration. 2) Venous crisis: the venous crisis is mainly manifested by the purple skin color at the end of the flap, decrease in the temperature of the flap, lighter or disappeared skin wrinkles, even occurrence of blisters on the surface of the flap, fullness of the finger pulp and increase in tension, fast capillary filling, arterial pulsation, rapid bleeding from fingertip incision, and the change of color from purple to bright red. Once a venous crisis occurs, the dressing should be first observed, and the tight dressing can be loosened.
The body position should be adjusted to make the flap pedicle loose. If the tension of the flap is too high, the affected limb can be raised, or a few suture lines away from the pedicle can be removed appropriately to relieve the tension of the flap [6-7]; (3) indicators for normal capillary measurement: when pressing the skin of the transplanted tissue with your fingers, the capillaries of the skin are emptied and the color turns white. After you withdraw your fingers, the capillaries recover and fill within a few seconds, generally no more than 2 seconds; (4) when arterial embolism occurs, the reflux is not smooth; when venous embolism occurs, the reflux speeds up early and slows down later; when arteriovenous embolization occurs, congestion remains in the capillaries and there is still reflux Hence, the determination of capillary filling is an important indicator to identify embolism and spasms; (5) tension: replanted fingers become full or elastic after blood transfusion; insufficient blood supply causes low tension while poor reflux leads to high tension; (6) small incision bleeding or bloodletting: when an arterial crisis occurs, there is no bright red blood flowing out of the incision; when a venous crisis occurs, the color of the blood bleeding from the small incision is dark purple [8]; (7) the calculation method of the incidence of vascular crisis: if any of the five situations: the color, skin tension, wrinkles, skin temperature and capillary filling measurement occurs and is not relieved after treatment with antispasmodic drugs, sympathetic nerve block and acupuncture, heating, small incision bloodletting, oxygen therapy, etc., it is counted as the occurrence of vascular crisis, The cases of vascular crisis to the total number of patients monitored ratio is the incidence of vascular crisis.

\subsection{Statistical Methods}

The data were analyzed using SPSS18.0. The measurement data were described by mean \pm standard deviation, and the pair $t$ test was used for comparison between groups. Enumeration data were described by frequency and percentage, and analyzed by $\chi^{2}$ test. The test level was $\alpha=0.05$, and $\mathrm{P}<0.05$ was considered statistically significant.

\section{Results}

\subsection{Comparison of the Occurrence of Vascular Crisis Between the Two Groups}

The incidence of postoperative vascular crisis in the observation group was significantly lower than that in the control group $(\mathrm{P}<0.05)$ (Table 1$)$.

Table 1. Comparison of the Occurrence of Vascular Crisis between the Two Groups.

\begin{tabular}{llll}
\hline Group & Cases & Combined injuries (blood vessel, nerve, tendon)/pure injury of blood vessel & Incidence of vascular crisis (\%) \\
\hline Control group & 35 & $15 / 20$ & $9(25.7 \%)$ \\
Observation group & 35 & $14 / 21$ & $2(5.7 \%)$ \\
$\chi^{2}$ & & 0.59 & 3.883 \\
$P$ & & 0.808 & 0.049 \\
\hline
\end{tabular}




\subsection{Comparison of Length of Stay and Duration of Wound Healing Between the Two Groups}

Local wound heals when local rosy skin, normal skin temperature, moderate skin tension, no capillary reflux obstacles, and clean wound surface can be observed.

The length of stay and duration of wound healing of the observation group were shorter than those of the control group, and the difference was statistically significant $(\mathrm{P}<0.05)$. Besides, the hospitalization cost of the observation group was statistically less than that of the control group $(\mathrm{P}<0.05)$, as shown in Table 2.

Table 2. Comparison of Length of Stay and Duration of Wound Healing between the Two Groups ( $\bar{x}_{ \pm}$).

\begin{tabular}{llll}
\hline Group & Cases & Length of stay (d) & Duration of wound healing (d) \\
\hline Control group & 35 & $30.1 \pm 6.6$ & $11.5 \pm 1.3$ \\
Observation group & 35 & $19.6 \pm 3.9$ & $8.5 \pm 0.8$ \\
$t$ & $/$ & 8.08 & 11.54 \\
$P$ & $/$ & 0.000 & 0.000 \\
\hline
\end{tabular}

\section{Discussion}

\subsection{Continuous Skin Temperature Monitoring is Conducive to Early Warning of Vascular Crisis After Hand Skin Soft Tissue Defect Repair}

Flap transplantation is employed to repair hand skin soft tissue defects, but postoperative vascular crisis is the primary reason for the failure of it [9]. By monitoring the temperature of the transplanted flap, the occurrence of vascular crisis can be predicted [10]. With high effectiveness and sensitivity, skin temperature monitor has a significant effect on nursing for flap repairing hand skin soft tissue defects. When using skin temperature monitoring device to continuously monitor the skin temperature of the transplanted flap, sudden rise or fall of the flap temperature suggest possible blood circulation crisis. A parallel curve shows that the skin temperature of the transplanted flap and the skin temperature of the healthy side are different by $\pm(0.5-2)^{\circ} \mathrm{C}$. A parallel change within $0^{\circ} \mathrm{C}$ indicates that the arteriovenous anastomotic opening is smooth and the blood circulation of the transplanted tissue is good. That the curve suddenly drops and the skin temperature of the transplanted flap and that of the healthy side suddenly differ by more than $3.00^{\circ} \mathrm{C}$ indicate arterial embolism, and surgical exploration should be performed immediately. A separation curve means that difference in the skin temperature of the transplanted flap and the healthy side gradually increases, generally reaching $3{ }^{\circ} \mathrm{C}$ after $24-48$ hours, which is caused by venous embolism. When these situations occur, nursing staff should report to the doctor for further measures to prevent arteriovenous crises, or even performing operation again to relieve vasospasm and its further deterioration. Skin temperature monitor can serve as an early warning of the change of the disease, so that advanced intervention can be taken to deal with the change in time, providing a more reliable guarantee for the survival of the flap. It can also provide an important reference for medical staff to detect the occurrence of blood circulation crisis.

\subsection{Blood Circulation of the Transplanted Flap Is Significant for Wound Healing and Prognosis Improvement}

The repair of hand skin soft tissue defects through surgery is the first step. As long as the blood vessels can be found under the microscope, anastomosis is given to alleviate the patient's psychological trauma and finger disability, improving the patient's quality of life [11]. However, the survival of the patient's flap after operation is the key to the success of the operation, and normal blood flow of transplanted skin flap tissue is an important part to ensure the survival of the flap. If there is a risk of necrosis of the transplanted flap and vascular occlusion [12], blood flow disorders or infections are prone to occur, which will lead to the failure of the surgery. It may require surgical treatment again, which will have a serious impact on the wound healing, length of stay and even the prognosis of the patient, causing physical and mental damage to the patient. The flap temperature is an important indicator to determine whether there is a vascular crisis or infection. Detection of the temperature change of the flap in time through continuous temperature monitoring in real time can help detect vascular crisis and infection. Measures to improve the blood flow of the transplanted flap and promote the survival of the flap can reduce the occurrence of postoperative complications, avoid reoperation due to flap necrosis, improve the success rate of the operation, and shorten the duration of wound healing and length of stay. It is of great significance for improving the prognosis of patients.

\section{Conclusions}

The skin temperature monitor is effective, sensitive and easy to operate. Skin temperature monitoring has a significant effect on the repair of skin soft tissue defects through flap transplantation. Continuous skin temperature monitoring after skin flap transplantation can detect the temperature change of the transplanted flap early, which is helpful for medical staff to take timely measures to reduce the occurrence of vascular crisis, ensure the survival of the flap, and improve the prognosis of the patient. It is thus worthy of clinical promotion 
and application.

\section{References}

[1] Xie Wenbin, Wang Haiwen, Jiang Xinming, et al. "Flow-through anterolateral thigh perforator flap for the treatment of skin and soft tissue defects of palm with circulatory disorder" [J]. Chinese Journal of Clinical Anatomy, 2016, 34 (1): 24-27.

[2] Guo Jia, Liu Yong, Liu Xiaohui, et al. "Iliac Flap Combined with Anterolateral Thigh Flap for Repair of First Metatarsal Bone and Large Skin Defect" [J]. Chinese Journal of Reparative and Reconstructive Surgery, 2016, 34 (11): 1400-1403.

[3] Heredero S, Solivera J, Blas García, et al. Osteomyocutaneous peroneal artery perforator flap for reconstruction of the skull base [J]. British Journal of Oral \& Maxillofacial Surgery, 2016, 54 (1): 99-101.

[4] Bozso S J, Grant A, Iglesias I, et al. Minimally Invasive Periareolar Approach to Unroofed Coronary Sinus Atrial Septal Defect Repair [J]. The Annals of Thoracic Surgery, 2016, 102 (3): e223-e225.

[5] Gao Xiaoyan, Yin Fang, Cao Jianhua, He Yuying, Wang Xiaoshuang, An Yanjing, Shen Jie. Jishuitan Hand Surgery Nursing and Rehabilitation [M]. People's Medical Publishing House Co., LTD, 2015: 126.
[6] Zhou Yang, Cheng Xianghong. A Practical Book for Hospital Orthopedics (Surgery) Clinical Nursing, Health Education and Nursing Rounds [M], first edition. People's Medical Publishing House Co., LTD, 2019: 246.

[7] Peng Yamei, Zhu Yanli. "Skin Temperature Monitoring on Nursing after Hand Flap Transplantation" [J]. Journal of Practical Hand Surgery, 2009, 23 (3): 191-192.

[8] Li Lezhi, Lu Qian. Surgical Nursing [M], fifth edition. People's Medical Publishing House Co., LTD, 2014: 157-158.

[9] Fu Yuhong, Mo Lan, Zhou Mingxia. "Ultra-Microsurgery Technique in Clinical Nursing of Replantation of Finger Pulp Tissue Block" [J]. Chinese Journal of Microsurgery, 2016, 39 (4): 410-411.

[10] Chimeric Anterolateral Thigh Flap for Total Thoracic Esophageal Reconstruction [J]. The Annals of Thoracic Surgery, 2016, 101 (1): 338-342.

[11] Song Jun, Guo Mingbo, Zhang Jingliang, et al. "The Replantation of Penetrating Severed Finger with Disconnected Micro Tissue Blocks in 16 Cases" [J]. Chinese Journal of Microsurgery, 2019, 42 (6): 533-535.

[12] Wei Yitao, Mei Xiongjun, Wu Renjuan, et al. "Clinical Study of Skin Grafting in Small Wounds with Anastomotic Vascular Exposure: Report of 16 Cases" [J]. Chinese Journal of Microsurgery, 2019, 42 (6): 536-539. 